\title{
Effects of the study design and analysis on the spatial community structure detected by multiscale ordination
}

Post-print/Accepted manuscript

Barbara M. Schlup

Helene H. Wagner

Schlup, B. M. and Wagner, H. H. (2008), Effects of study design and analysis on the spatial community structure detected by multiscale ordination. Journal of Vegetation Science, 19: 621-632. doi: $10.3170 / 2008-8-18421$

This is the peer reviewed version of the following article: Schlup, B. M. and Wagner, H. H. (2008), Effects of study design and analysis on the spatial community structure detected by multiscale ordination. Journal of Vegetation Science, 19: 621-632, which has been published in final form at doi:10.3170/2008-8-18421 This article may be used for non-commercial purposes in accordance with Wiley Terms and Conditions for SelfArchiving.

\section{HOW TO CITE TSPACE ITEMS}

Always cite the published version, so the author(s) will receive recognition through services that track citation counts, e.g. Scopus. If you need to cite the page number of the TSpace version (original manuscript or accepted manuscript) because you cannot access the published version, then cite the TSpace version in addition to the published version using the permanent URI (handle) found on the record page. 


\section{Effects of the study design and analysis on the spatial community}

\section{2 structure detected by multiscale ordination}

3

4 Barbara M. Schlup ${ }^{1,2 *}$ \& Helene H. Wagner ${ }^{1,3}$

$5 \quad{ }^{1}$ WSL Swiss Federal Research Institute, CH-8903 Birmensdorf, Switzerland;

$6 \quad{ }^{2}$ Forest Ecology, Department of Environmental Sciences, Swiss Federal Institute of

7 Technology Zurich, CH-8092 Zurich, Switzerland;

$8{ }^{3}$ Department of Ecology and Evolutionary Biology, University of Toronto, Mississauga, ON,

9 Canada L5L 1C6

10

11

12

13

$14 *$ Corresponding author:

15 Barbara M. Schlup,

16 WSL Swiss Federal Research Institute,

$17 \quad$ CH-8903 Birmensdorf, Switzerland

18 Phone: +41-1-739-2807,

19 Fax: +41-1-739-2215,

20 E-mail: barbara.schlup@wsl.ch 


\section{Abstract}

2 Question: What is the effect of different aspects of data collection and analysis on the spatial

3 partitioning of ordination results by multiscale ordination?

4 Location: Heterogeneous pasture at Marchairuz in the Jura mountains, Switzerland. Mixed

5 hardwood-pine forest in Oostings Natural Area, NC, USA.

6 Methods: We evaluated the efficiency of different sampling designs for identifying the spatial

7 structure of plant communities and analyzed two data sets with multiscale ordination. We

8 compared the effects of quadrat size, the number of species included in the analysis, data type,

9 detrending and ordination method on the shape and precision of the community variogram

10 summarizing spatial community structure.

11 Results: A three-block sampling design provided a more even distribution of the number of

12 pairs of observation per distance class than random, transect or grid designs. The precision of variogram estimates depended more strongly on the number of species than on the number of quadrats. In contrary, the choice of data type (abundance transformation) had little influence on the shape of the variogram. Detrending reduced the range of spatial autocorrelation. An increase in quadrat size resulted in a smoother variogram and stronger spatial autocorrelation. Principal components analysis (PCA) and redundancy analysis (RDA) resulted in a larger range of spatial autocorrelation than using correspondence analysis (CA) and canonical correspondence analysis (CCA), but the shape of the variograms was rather similar.

Conclusion: Random samples as well as transects and regular grids may not be efficient sampling designs for spatial analysis of community structure. While the number of species considered strongly affects the precision of a community variogram, its shape depends on the

23 size of the sampling units. Earlier studies may have overestimated the spatial scale of internal organization in plant communities. 


\section{Keywords}

Spatial pattern; variogram; gradient analysis; spatial ecology.

\section{Abbreviations}

(1)

$$
\mathrm{MSO}=\text { Multiscale ordination } \text { CA = Correspondence analysis } ; \mathrm{CCA}=\text { Canonical }
$$
correspondence analysis; PCA = Principal components analysis; RDA = Redundancy analysis; $\mathrm{CV}=$ Coefficient of variation; $\mathrm{BQV}=$ Blocked-quadrat variance .

\section{Introduction}

Plant community ecology aims at identifying and interpreting the structure of species assemblages. Community structure is commonly evaluated in terms of gradients in species composition, using either presence/absence or abundance data. Legendre (1993) distinguished between "true gradients" in species composition, which are induced by environmental gradients, and "false gradients", which may arise even in the absence of environmental heterogeneity as a result of biotic interactions within the community. When mapped into geographic space, both true and false gradients may form distinct spatial patterns. It has even been suggested that different ecological processes create distinct spatial patterns, so that specific processes could be identified from their spatial signature (Seabloom et al. 2005). Hence, spatial analysis of community structure is of direct scientific interest, because spatial structures may be critical for identifying and understanding the underlying ecological processes (Dale 1999).

Spatial pattern analysis has a long tradition in plant ecology, and a wide variety of methods is available as reviewed by Dale (1999). Blocked-quadrat variance methods (BQV), which date back to Greig-Smith (1952) and Kershaw (1957), allow for the identification of spatial patterns from transects or grids of contiguous sampling quadrats. Adjacent quadrats are 
1 aggregated to blocks of increasing size (Dale 1999) and the variance amongst blocks is plotted

2 against block size so that a peak of variance can indicate the size of a regular pattern. These

3 methods are used routinely for summarizing the spatial structure of single species and pairs of

4 species (Fortin 1999b). Under the name of multiscale ordination, Noy-Meir and Anderson

5 (1971) and Ver Hoef and Glenn-Lewin (1989) extended BQV to the analysis of multi-species

6 patterns. Nevertheless, spatial pattern analysis remained little integrated with multivariate

7 community analysis such as classification and ordination, the non-spatial standard methods for

8 analyzing community structure.

This lack of integration may reflect important conceptual differences beyond the

obvious question of explicit versus implicit treatment of geographic space. Classical

11 vegetation science focuses mostly on true gradients, i.e., the differentiation of plant

communities along environmental gradients using either a discrete (classification) or a continuous (ordination) vegetation paradigm. By spacing quadrats in a random or regular fashion, observations are implicitly assumed to be spatially independent. Spatial pattern analysis, on the other hand, analyzes the spatial structure of false gradients reflecting internal community organization in an assumedly homogeneous environment (Table 1).

It is increasingly recognized, however, that environmental heterogeneity may occur at several scales (Wiens 1989; Dutilleul 1993). Furthermore, a random sample does not guarantee the spatial independence of observations (Fortin et al. 1989), and spatial autocorrelation is a common phenomenon in ecological data (Legendre 1993). Some BQV methods have been developed to account for environmental heterogeneity (detrending), e.g. the three-term local quadrat variance (cf. Dale 1999). This method does not rely on measured environmental predictors, but removes apparent trend by the local fitting of a linear trend surface to the response variable. In addition, the spatial autocorrelation of community similarity can be used to determine the distance beyond which observations may be assumed 
1 to be independent, providing a minimum distance for the spacing of quadrats in random or

2 regular sampling designs for the analysis of true gradients (Dungan et al. 2002). This can be

3 achieved, e.g., by plotting a "dissimilogram" (Mistral et al. 2000), i.e. a plot of dissimilarity

4 against distance, or univariate empirical variogram, i.e. a plot of the semivariance against

5 distance, of the scores of the first ordination axis (Palmer 1987). In the absence of true

6 gradients, such variogram-type plots are expected to show an initial increase up to the range,

7 i.e. the distance up to which signifcant positive autocorrelation can be found, beyond which a constant value (sill) should be reached, indicating the dissimilarity or variance among spatially independent observations. In a spatially heterogeneous environment, however, these methods are likely to overestimate the distance over which the response of species to their environment is spatially autocorrelated, because they do not distinguish between the spatial structure due to true and false gradients (Wagner and Fortin 2005). $(2003 ; 2004)$ provides a mathematical integration of ordination with spatial analysis using variograms (see Appendix). The analysis of spatial structure is based on the geographic distance between pairs of quadrats, rather than the aggregation of contiguous quadrats, so that it can be applied to a wide range of sampling designs. The variance-covariance matrix is partitioned into a set of distance-dependent matrices, which together form the variogram matrix (Wagner 2003). This approach effectively provides distance-dependent estimates for any ordination parameter, such as total, constrained and residual variance, eigenvalues for ordination axes, or the correlation between species composition and site factors. The variogram matrix has a geostatistical interpretation, as it contains the empirical variograms of all species and cross-variograms of all pairs of species. The variogram of the total variance in the species data (community variogram) can thus be decomposed into the sum of the species variograms. This allows spatial analysis at the level of individual species, pairs of species, 
1 groups of species, or the entire community, as well as of different component patterns in terms

2 of the variance represented by specific ordination axes. The method extends to constrained 3 ordination (direct MSO) using redundancy analysis or canonical correspondence analysis

4 (Wagner 2004) and other ordination methods (Couteron and Ollier 2005). Direct MSO results

5 in a spatial partitioning of the variance explained by environmental predictors (true gradients)

6 and the residual variance (assumed to reflect false gradients), and provides a diagnostic tool

7 for checking the assumptions of homogeneity and independence (Wagner 2004). Hence, environmental heterogeneity represented by measured predictor variables can be accounted for while assessing the range of residual spatial autocorrelation attributed to processes within the community. This is important because it is the residual autocorrelation, rather than the total spatial structure in the response data, that violates the assumption of independence in the estimation of the species-environment relationship (Wagner and Fortin 2005). The geostatistical version of MSO has several advantages over BQV methods, including the possibility of accounting for environmental heterogeneity measured by predictor variables, compatibility with a wide range of ordination methods (Wagner 2004; Couteron and Ollier 2005), the combined analysis of patterns at species and community level, and the

17 flexibility of the sampling design. Spatial statistics such as Moran's I (Moran 1950) also provide a test for spatial autocorrelation and can be applied either to the original species data 19 or the residual values, but they need to be calculated separately and are not directly related to ordination parameters. Due to its flexibility and the integration of spatial and non-spatial analysis, multiscale ordination has a great potential for the analysis and interpretation of spatial structure in plant communities. However, little is known about the robustness of the method with respect to the sampling design and other aspects of data collection and analysis that may affect the detection of spatial structures in plant communities, as reviewed below. 
Sampling design: Legendre et al. (2004) provide advice for ecologists on the appropriate sampling design and experimental analysis when the goal is to assess treatment effects accounting for spatial autocorrelation such as induced by environmental heterogeneity. The ecological literature, however, offers little guidance on sampling design for spatial analysis beyond the classical designs of 1-dimensional transects and 2-dimensional regular grids (Fig. 1). Fortin and Dale (2005) discussed the topic mostly from the point of avoiding directional bias but do not explicitly treat the aspect of precision in estimates of spatial structure. Empirical variogram estimates for each distance class tend to fluctuate around their expected values, resulting in considerable scatter around an ideal smooth variogram curve. The interpretability of an empirical variogram depends on its smoothness and thus on the precision with which the semivariance of each distance class can be estimated. This precision is expected to increase with the number of pairs of observations in the distance class. The number of pairs tends to vary with sample size and the definition of distance classes (lag), but also with the spatial configuration of the sample. Hence, we need to know which types of sampling designs are most efficient for identifying the spatial structure of plant communities. Sample size and species number: Fortin and Dale (2005) recommend a sample size of 30 for testing for the presence of spatial autocorrelation, and a sample of 100 for estimating spatial structure, such as in variogram or correlogram analysis. This is because the precision of these distance-dependent estimates depends on the number of pairs per distance class. Epperson (2003) recommends calculating Moran's I correlograms from at least 200 pairs per distance class. These rules, however, may not be applicable to the multivariate case.

Data type: Plant community data typically are not true counts of individuals because of the difficulty to define an individual in the field. Furthermore, for many research objectives on vegetation communities not the number of individuals is of primary interest, but the space occupied by each plant species, so that vegetation data are often collected using an ordinal 
1 abundance scale that reflects differences in species cover. These data may be transformed for

2 subsequent analysis depending on the research objective, with the assignment of class mean

3 percent cover values or reduction to presence-absence data as the two extremes (Gauch 1992).

4 The question here is how sensitive the spatial analysis of community structure is to the choice

5 of data type, i.e., abundance transformation.

Detrending: Many studies of spatial structure in plant communities found spatial autocorrelation over large distances (e.g., Jonsson and Moen 1998; Mistral et al. 2000; Scheller and Mladenoff 2002; Augustine 2003). While these analyses were based on the original species data, it is often recommended in the geostatistical literature that data are detrended before analysis, either by fitting a linear or polynomial trend surface model or by performing spatial analysis on the residuals of a regression-type model (cf. Wagner and Fortin 2005). Here we aim at investigating if the range of spatial autocorrelation may be reduced after accounting for environmental heterogeneity.

Quadrat size: The size of the sampling quadrat may have an important effect on the observed spatial patterns, a problem known as the modifiable areal unit problem (Openshaw 1984; Jelinski and Wu 1996; Dungan et al. 2002). Community ecology has made little attempts at formalizing the effect of quadrat size beyond the species-area relationship. A notable exception are studies based on the information-theoretic approach by Juhász-Nagy and Podani (1983), who defined several diversity measures that have been used for scaling studies. The approach can be used to determine an appropriate quadrat size (Juhász-Nagy 1976; Juhász-Nagy 1984; Podani 1984), and Bartha et al. (1998) suggested that the scale-dependent maxima of two diversity measures (compositional diversity and coenological diversity) can be interpreted in terms of dominant processes shaping a community. Palmer and White (1994) found that the degree to which the number of new species encountered in a second quadrat but not in the first depended on the distance between quadrats increased with quadrat size. It is 
1 important to know, therefore, whether and how the size of the sampling unit affects for

2 instance the observed range of autocorrelation.

Ordination method: Principal components analysis (PCA) and redundancy analysis

4 (RDA) are based on a variance-covariance or a correlation matrix of species variables, while

5 correspondence analysis (CA) and canonical correspondence analysis (CCA) calculate this

6 matrix from deviations from expected values used in the chi-square statistic (Legendre and

7 Legendre 1998). It is unclear to what degree the observed spatial pattern detected by MSO,

8 e.g., the range of significant spatial autocorrelation, depends on the ordination method used.

The purpose of this paper is to investigate the consequences of different aspects of

10 data collection and analysis on the detection of spatial structure in plant communities using

11 multiscale ordination. The paper focuses on the community variogram, the spatial

12 decomposition of the overall variance in the plant community. We use a new dataset from a

13 pasture at Marchairuz in the Swiss Jura Mountains and well-known data from Oosting Nature

14 Reserve (Reed et al. 1993, Palmer and White 1994, Palmer 1995, Jonsson and Moen 1998,

15 Wagner 2003), to analyze the robustness of variograms towards changes in the spatial

16 configuration of the sampling design, sample size and species number, data type, quadrat size,

17 detrending and ordination method. Rather than providing an in-depth analysis of a single

18 aspect based on extensive simulation experiments, we aim at comparing the different effects

19 using real data in order to identify the most relevant factors as a focus for future research.

20 Methods

\section{Marchairuz data}

The data set included presence-absence and abundance data (shooted frequency) of

23 plant species from a heterogeneous pasture at Marchairuz in the Jura Mountains of 
1 northwestern Switzerland. The sampling design was equivalent to design D in Figure 1 (for

2 further description of the data see Appendix).

\section{Oosting data}

We used previously published data from a mixed hardwood-pine forest in North Carolina (Reed et al. 1993, Palmer and White 1994, Palmer 1995, Jonsson and Moen 1998, Wagner 2004). The study plot was designed specifically to address questions related to scale and spatial pattern. With the choice of different quadrat sizes, different resolutions for the analysis can be obtained. Besides the dataset containing the entire grid, we used presenceabsence data of the module SM3 that had previously been analyzed by Wagner (2003) (for further description of the data see Appendix).

\section{Effect of the sampling design}

We compared four sampling designs that differed in the arrangement of the sampling units, while keeping sample size constant. We were interested in the number of pairs of observations per distance class, where each pair "ab" and "ba" is only counted once, and the maximum extent for interpretation, which we defined as half the maximum distance between observations. Beyond this distance, not all pairs will contribute to the class-wise estimate, and the number of pairs may decrease rapidly. As sampling designs we used a transect (Figure $1 \mathrm{~A}$ ), a single block (Figure $1 \mathrm{~B}$ ), a random sample (Figure $1 \mathrm{C}$ ) and a 3-blocks design (Figure $1 \mathrm{D}$ ). With this last design, we aimed at achieving an even number of pairs of observations per distance class, a minimum of approximately 100 pairs per class, an interpretable extent of $8-10 \mathrm{~m}$, and the possibility for aggregating observations to blocks of two or more quadrats. For each sampling design, we calculated the number of pairs of quadrats in each distance class, setting the lag distance to quadrat length. 


\section{Effect of sample size and species number}

We compared the effects of sample size and of the number of species on variogram smoothness, or precision, by resampling quadrats and species from the Marchairuz data set, using abundance data. The effect of sample size was assessed from 100 subsamples of each sample size of 12,24 , and $36(25 \%, 50 \%$, and $75 \%$ of total sample), using all 67 species. To assess the effect of species number, 100 replicate subsamples each of 5, 10, 20 and 40 species were taken, using all 48 quadrats.

For each subsample, we calculated an empirical variogram from the abundance data (square-root transformed ranks, see Appendix) using a lag distance of $1 \mathrm{~m}$. The range of positive spatial autocorrelation was determined for each variogram with a one-sided permutation test (100 replications) applying a progressive Bonferroni correction (Hewitt et al. 1997; Legendre and Legendre 1998). For each repetition, the sample coordinates were permuted randomly and a community variogram was calculated. P-values were obtained for each distance class by counting the proportion of semivariance values smaller than the observed. The procedure of the progressive Bonferroni correction is fundamentally different from the sequential Bonferroni correction (Holm 1979) that adjusts all p-values in a table equally by dividing the significance level alpha by the number of tests, resulting in a large loss of statistical power (e.g., Moran 2003). With the progressive Bonferroni approach, the significance level $\alpha$ decreases as one proceeds from the smallest to the larger distance classes of the variogram with $\alpha_{k}=0.05 / k$ for the $k^{\text {th }}$ distance class, up to the first non-significant distance class. This correction is appropriate when the interest is primarily in detecting positive spatial autocorrelation in the first distance classes, namely for assessing the range of spatial autocorrelation due to a stationary spatial process (Legendre and Legendre 1998). 
1 non-significant distance classes, based on the above test with progressive Bonferroni

2 correction, as all these values have the same expected value. The analysis was restricted to a

3 maximum lag of 8 m., i.e., up to half the maximum distance between quadrats. The mean $\mathrm{CV}$

4 and its $95 \%$ confidence interval were estimated from the 100 replicate subsamples for each

$5 \quad$ sample size and species number.

6 Effect of data type

The effect of data type was investigated with the Marchairuz data set, as abundance data were needed. We analyzed the spatial structure with four different transformations of the

9 original data, including presence-absence, square-root transformed ranks, original ranks, and raw abundance data in terms of mean percent cover of abundance classes. For each data type,

11 a multiscale ordination on the basis of CA was calculated. To ensure a minimum number of 100 pairs of quadrats for each distance class up to $10 \mathrm{~m}$, the distance classes of $4 \mathrm{~m}$ and $5 \mathrm{~m}$ were pooled. As the resulting variograms of total variance showed no indication of a trend, we did not include any environmental predictors. To make the variograms directly comparable, each variogram was standardized by dividing it by the total variance of the data set. For the assessment of the range of positive spatial autocorrelation see above.

\section{Effect of detrending}

With the entire Oosting dataset of 256 quadrats and the SM3 subset, a direct MSO on the basis of PCA and RDA was calculated, applying a lag distance of $1 \mathrm{~m}$. Direct MSO results

20 in a separate variogram for the variance explained by environmental variables and the residual

21 variance, allowing for the comparison of the range of spatial autocorrelation before and after detrending. For the assessment of the range of positive spatial autocorrelation see above. 
For the entire Oosting dataset, we included elevation and soil factors as

2 environmental variables. With the 24 soil factors of the data set, we made the same

3 transformations as recommended by Reed et al. (1993) to increase normality and reduce the

4 influence of outliers. For the SM3 subset, the soil variables were not available at the cell level.

5 Wagner (2003) found a strong correlation of $r=0.73$ between the first PCA axis and a linear

6 interpolation of elevation based on values measured at the corners of the SM3 module. Hence,

7 we used interpolated elevation (Wagner 2003) and the first PCA axis alternatively as

8 environmental predictors in direct MSO with RDA, retaining in both cases only the residuals

9 for further analysis.

\section{Effect of quadrat size}

A PCA of the presence-absence data was calculated for four different quadrat sizes of the SM3 subset of the Oosting data set. Again we used interpolated elevation (Wagner 2003, see above) as environmental predictor in direct MSO with RDA, applying a lag distance of 1 $\mathrm{m}$. For the assessment of the range of positive spatial autocorrelation see above.

\section{Effect of the ordination method}

To assess the effect of different ordination methods on the observed spatial pattern, we repeated the analysis of both data sets using PCA/RDA and CA/CCA.

All calculations were performed in R (Ihaka and Gentleman 1996) using the libraries “vegan" (URL: http://www.r-project.org) and "mso" (Wagner 2004). 


\section{Results}

\section{Effect of the sampling design}

The number of pairs of observations per distance class and the maximum extent for interpretation of a variogram varied with the spatial configuration of the four different sampling designs. The maximum extent of interpretation ranged from $4 \mathrm{~m}$ for the single block design to $23 \mathrm{~m}$ for the transect, with $8 \mathrm{~m}$ for the other two designs (Figure 2). The transect design (Figure $1 \mathrm{~A}$ ) resulted in a maximum of 47 observations per distance class (Figure $2 \mathrm{~A}$ ), while all other designs reached a minimum of 100 pairs per distance class. Whereas the single block (Figure $1 \mathrm{~B}$ ) and random design (Figure $1 \mathrm{C}$ ) had the lowest frequencies in the first three distance classes (Figure 2 B, C), the 3-blocks design (Figure 1 D) had the highest values in the first two distance classes (Figure 2 D). Pooling distance classes 4 and 5 reduced variability to the range of $92-126$ (Figure $2 \mathrm{E}$ ), with a minimum close to the targeted 100 pairs per distance class.

\section{Effect of sample size and species number}

Resampling species from the full Marchairuz data set indicated that the coefficient of variation $\mathrm{CV}$ of the variogram estimates decreased markedly with increasing number of species, suggesting an exponential relationship (Figure 3, left panel). This effect was comparable to a massive increase of sample size (Figure 3, right panel). For instance, the precision of variogram estimates based on a subsample of 20 out of 67 species (0.06) was similar to the precision reached with all species but only $24(50 \%)$ of the quadrats $(0.059)$.

\section{Effect of data type}

The analysis of the effect of data type, or transformation, on the shape of an empirical variogram of the Marchairuz data revealed little difference between presence-absence data and 
1 the square-root transformed rank data (Figure 4, left panel). In both cases, spatial

2 autocorrelation was significant for the first two distance classes, resulting in a range estimate

3 of $2 \mathrm{~m}$ using a lag distance of $1 \mathrm{~m}$. Even the use of untransformed ranks increased the

4 amplitude of the variogram very little and did not affect the estimate of the range of spatial

5 autocorrelation. However, the extreme case, where ranks were replaced by class mean cover

6 values, resulted in a considerable increase of the amplitude, combined with a tendency for

7 periodic behavior. Initial significant positive autocorrelation was restricted to the first distance

8 class with an estimated range of $1 \mathrm{~m}$.

\section{Effect of detrending}

The range of spatial autocorrelation was much shorter when explanatory variables

were included in the analysis. The variogram of the total variance based on the entire Oosting data set of 256 quadrats showed a range of significant spatial autocorrelation of almost $100 \mathrm{~m}$ and a slight increase of variance with distance, suggesting the presence of a trend (Figure 5, left panel). After accounting for soil factors and elevation, the range was reduced to $19 \mathrm{~m}$, and the residual variogram reached a sill.

On the basis of the SM3 data, the variogram of the total variance showed significant spatial autocorrelation up to a distance of $5 \mathrm{~m}$ (Figure 5, right panel). After accounting for interpolated elevation or the first axis of PCA, the range was reduced to $3 \mathrm{~m}$ and the residual variograms showed no increase with distance anymore.

\section{Effect of quadrat size}

The size of the sampling units affected the variance and the spatial pattern in the data. Based on PCA analysis, the total variance increased from 1.01, 1.60, 3.20 to 5.44 (in order of increasing quadrat size), accompanied by an increase in the strength of spatial 
1 autocorrelation (see Appendix, Figure A.1, top left). The residual variance after accounting for

2 interpolated elevation using RDA increased from $0.19,0.20,0.36$ to 0.60 (not shown). Based

3 on CA analysis, the total variance varied between 7.69, 11.14, 6.98 and 4.28 (see Appendix,

4 Figure A.1, top right). The residual variance using CCA decreased from 1.00, 0.74, 0.39 to

50.22 (not shown).

6 The standardized residual variograms based on both RDA and CCA suggested

7 greater strength for the larger quadrat sizes, i.e. a lower nugget effect $(0.81$ for the $1 \mathrm{~m}$

8 resolution in RDA, 0.87 in $\mathrm{CCA}$ ), indicating that a lower proportion of the variance remained

9 unexplained, and a larger range of spatial autocorrelation (see Appendix, Figure A.1, bottom

10 right and left). The variograms for the smallest quadrat sizes were rather erratic, whereas the

11 variograms for the two largest quadrat sizes were quite smooth. For all methods, the range

12 estimate in terms of the number of distance classes with significant autocorrelation increased

13 with quadrat size (see Appendix, Figure A.2). Additional analyses suggested that this could be explained by sample size for the smallest distance class only (results not shown).

\section{Effect of the ordination method}

The PCA (Figure 4, left panel) and CA results (Figure 4, right panel) for the

Marchairuz data showed very little difference between the patterns revealed by the two ordination methods as compared to the difference between data types. The analysis of the Oosting data set SM3 for four different quadrat sizes, however, showed that the way in which total variance changed with quadrat size differed markedly between ordination methods (see Appendix, Figure A.1, top). While the total variance in the Oosting SM3 data set increased with quadrat size in PCA, CA showed a maximum for the $0.25 \mathrm{~m}$ resolution. However, this had little effect on the standardized variograms of the total variance (not shown) and the residual variance (see Appendix, Figure A.1, bottom). The nugget effect gradually decreased 
1 over quadrat size in both ordination variograms (see above), with the exception of the smallest

2 quadrat size, but the shape of the variograms and the relative magnitude of autocorrelation

3 across quadrat size were rather similar.

The number of distance classes with significant spatial autocorrelation in the Oosting data set SM3 was higher for PCA than for RDA and for CA than for CCA respectively (see

6 Appendix, Figure A.2). Accounting for interpolated elevation with RDA and CCA reduced 7 this number for both methods.

\section{Discussion}

\section{Effect of the sampling design}

The problem of an optimal spatial sampling design has many facets (e.g. JuhászNagy and Podani 1983; Kenkel et al. 1989), as reviewed by Fortin and Dale (2005). Here, we focused on two aspects. First, the smoothness of the variogram, i.e., the precision of variance estimates, which is a function of the number of pairs of observations per distance class. Second, the maximum distance for interpretation, defined as half the maximum extent, where larger distance classes are calculated from observations near the border of the study area only. The two most common sampling designs for spatial analysis, transects and grids, each optimize one of these two properties, i.e. the number of pairs of observations and the maximum extent (see Figure 2). However, there seems to be a trade-off between these aspects, so that intermediate designs may be more appropriate in many cases. A transect results in very low numbers of pairs per distance class, whereas a grid of contiguous quadrats allows the assessment of spatial structure for very short distances only. Random samples typically have few observations in the first few distance classes, which usually are of the greatest interest for the assessment of spatial autocorrelation. In our example, a simple design involving three 
1 blocks of contiguous samples provided three times as many pairs for the first distance class

2 than a random sample designed to allow interpretation to the same maximum distance. In

3 addition, the number of pairs per distance class was more balanced, and fewer pairs were

4 "lost" in distance classes beyond the interpretation limit. Low numbers of pairs at intermediate

5 distances may be avoided by pooling of neighboring classes. This is not necessary, but

6 increases the minimum number of pairs per distance class and thus the smoothness of the

7 variogram.

\section{Effect of sample size and species number}

We found that the precision of variogram estimates was primarily dependent on species number, i.e., whether all species or only subsamples of species were included, then sample size, especially for medium sized samples. The higher influence of species number than sample size makes it difficult to define a simple recommendation for the number of pairs per distance class, as it will depend not only on the required precision, but also on the analysis level. Specifically, the comparison of spatial structure among species or groups of species will require much more data than an assessment of the overall range of autocorrelation in a community.

Several alternative explanations for this effect of species number are possible. First, while each species variogram is noisy, this noise may be averaged out when many species variables are aggregated. This may be especially true in the case of negative associations between species. Second, the effect may be due to the omission of certain species, so that it would depend on the percentage of the species of a community included in the analysis, rather than on the absolute number of species. Third, the effect might be related to the properties of the species included in the analysis, for instance if infrequent species show stronger patchiness (effect of species incidence). 


\section{Effect of data type}

The community variograms in our study were surprisingly robust towards the data type. With the exception of mean cover, which gives a very high weight for frequent species, all abundance transformations of cover estimates resulted in a similar variogram shape and range of significant autocorrelation. This suggests that for comparable data sets with the aim to assess the range of spatial autocorrelation in species composition, it may suffice to collect presence-absence data, as long as the hypothesis concerns species composition rather than biomass. Our result is consistent with the findings by Fortin (1997) in the context of boundary detection, whereas Podani and Csontos (2006) found different shapes in variograms with different data types (presence-absence data and percentage cover).

\section{Effect of detrending}

Accounting for environmental heterogeneity reduced the range of significant spatial autocorrelation to a few meters. The first PCA ordination axis or the interpolated elevation can be valuable variables to represent lacking environmental factors. Here, they explained enough variance to remove a trend in the data and therefore allowed for the interpretation of the variogram.

\section{Effect of quadrat size}

The modifiable areal unit problem states that quadrat size may have en effect on the estimated variance (Openshaw 1984, Dungan et al. 2002). Using PCA, we found that both the total variance and the slope of the variogram increased with quadrat size. This corresponds to the results of the original analysis of Palmer and White (1994), based on a plot of the number of species present in a second quadrat but not in the first. Also Podani and Csontos (2006) 
1 found similar results with presence-absence data of a plant community, analyzing the effect of

2 quadrat size upon the estimation of spatial autocorrelation.

When using CA, however, we found that total variance (or inertia) did not change

4 monotonically with quadrat size but peaked at the second smallest quadrat size. This

5 behaviour is consistent with the information-theoretic approach by Juhász-Nagy and Podani

6 (1983), where the variance is expected to be at a maximum with a certain quadrat size.

A comparison of standardized variograms showed the same effect on variogram shape for both ordination methods. An increase in quadrat size resulted in a smoother variogram and a stronger spatial autocorrelation. This was true for the variograms of the total variance and for the residual variograms after accounting for interpolated elevation. This is

11 compatible with results from univariate analysis, where Fortin (1999a) found that the magnitude of autocorrelation in species abundance data increases with quadrat size, eventually

13 reaching a plateau or even declining with very large quadrat sizes as more environmental 14 heterogeneity is included within quadrats.

\section{Effect of the ordination method}

Our results suggest that the choice of ordination method had only a small effect on the represented spatial structure of the community. Although the way in which the total variance changed with quadrat size differed markedly between ordination methods, corresponding to the results of Otypková and Chetry (2006), this had little effect on the standardized variograms of the total and residual variance. There was a tendency for stronger autocorrelation extending over a larger number of distance classes when using PCA as compared to CA. These differences may be magnified when direct MSO (with larger gradients) is used to account for environmental heterogeneity, where the model is sensitive to 
1 the response shape. However, if the method is implemented correctly, or if the environment is

2 homogeneous, the details of the ordination method may play a minor role.

More research is needed to develop reliable ways of detecting model specification

\section{Conclusions}

While sample size is the main factor affecting the precision of estimates in nonspatial statistics, this does not seem to hold for the spatial analysis of multispecies assemblage data in a geostatistical framework. First, it is the number of pairs per distance class that determines variogram smoothness, i.e., the precision of class-wise variogram estimates. For a given sample size, the distribution of pairs of sampling units depends strongly on the spatial configuration of the sampling units, and in most cases neither a transect or grid design nor a simple random sample will provide optimal results, but the 3-block sampling design (Fig. 1 D) may be most efficient. The sampling design should be optimized based on the targeted lag distance, the maximum distance for interpretation, and the number of pairs per distance class. Second, the precision of the multivariate community variogram depends to a large degree on the number of species analyzed. While further research is needed to understand the mechanism underlying this effect, it is clear that the analysis of single-species patterns or the comparison of spatial structure between groups of species will require a considerably larger sample size than the community-level assessment of spatial structure.

Third, quadrat size has a systematic effect on the intensity of the pattern, i.e., the strength and the range of significant autocorrelation. We recommend nesting multiple quadrat 
1 sizes as in Palmer and White (1994), so that the sensitivity of a specific result to quadrat size

2 can be assessed. However, very small quadrats may provide little information as they are more

3 likely to be empty, thus effectively reducing sample size.

Last but not least, environmental heterogeneity often induces spatial structure that

reaches beyond the scale of internal organization in a community. Hence, accounting for

6 environmental heterogeneity will often reduce the observed scale of patchiness. Earlier studies

7 that failed to account for environmental heterogeneity may have overestimated the spatial

8 scale of internal organization in plant communities (cf. Wagner and Fortin 2005).

\section{Acknowledgments}

This research is part of a project funded by the Swiss National Science Foundation

(SNF) under the NCCR Plant Survival. We thank Mike Palmer, Oklahoma State University,

12 for providing the Oosting data sets, and Harald Bugmann, Swiss Federal Institute of

13 Technology (ETH Zürich), for the supervision of the diploma thesis from which the

14 publication evolved and for his valuable comments on the manuscript. We also thank Valério

Pillar and two anonymous reviewers for their helpful comments.

\section{References}

Augustine, D. J. 2003. Spatial heterogeneity in the herbaceous layer of a semi-arid savanna ecosystem. Plant Ecology 167: 319-332.

Bartha, S., Czárán, T. and Podani, J. 1998. Exploring plant community dynamics in abstract coenostate spaces. Abstracta Botanica 22:49-66.

Braun Blanquet, J. 1964. Pflanzensoziologie: Grundzüge der Vegetationskunde. $3^{\text {rd }}$ German ed. Springer Verlag, Wien. 
1 Camiz, S. 1991. Reflections on Spaces Relationships in Ecological Data Analysis: Effects, Problems, Possible Solutions. Coenoses 6(1): 3-13.

3 Camiz, S. 1993. Computer Assisted Procedures for Structuring Community Data. Coenoses 8(2): 97-104.

Couteron, P. \& Ollier, S. 2005. A generalized, variogram-based framework for multi-scale ordination. Ecology 86(4): 828-834.

Dale, M. R. T. 1999. Spatial Pattern Analysis in Plant Ecology. Cambridge University Press, Cambridge.

Dierschke, H. 1994. Pflanzensoziologie - Grundlagen und Methoden. Ulmer Verlag, Stuttgart.

Dungan, J. L., Perry, J. N., Dale, M. R. T., Legendre, P., Citron-Pousty, S., Fortin, M.-J., Jakomulska, A., Miriti, M. \& Rosenberg, M. S. 2002. A balanced view of scale in spatial statistical analysis. Ecography 25(5): 626-640.

Dutilleul, P. 1993. Spatial heterogeneity and the design of ecological field experiments. Ecology 74(6): 1646-1658.

Epperson, B. K. 2003. Geographical Genetics. Princeton, Princeton University Press.

Fortin, M.-J. 1997. Effects of data types on vegetation boundary delineation. Canadian Journal of Forest Research-Revue Canadienne De Recherche Forestiere 27(11): 1851-1858.

Fortin, M.-J. 1999a. Effects of sampling unit resolution on the estimation of spatial autocorrelation. Ecoscience 6(4): 636-641. 
1 Fortin, M.-J. 1999b. Spatial statistics in landscape ecology. In: Klopatek, J. M. \& Gardner, R.

H. (eds.). Landscape ecological analysis: issues and applications. pp. 253-279. Springer-Verlag, New York, USA.

Fortin, M.-J., Drapeau, P. \& Legendre, P. 1989. Spatial autocorrelation and sampling design in plant ecology. Vegetatio 83(1-2): 209-222.

Fortin, M.-J. \& Dale, M. 2005. Spatial Analysis: A Guide for Ecologists. Cambridge, Cambridge University Press.

Gauch, H. G. 1982. Multivariate analysis in community ecology. Cambridge University Press.

Greig-Smith, P. 1952. The use of random and contiguous quadrats in the study of structure in plant communities. Annals of Botany 16: 293-316.

Hewitt, J. E., Legendre, P., McArdle, B. H., Thrush, S. F., Bellehumeur, C. \& Lawrie, S. M. 1997. Identifying relationships between adult and juvenile bivalves at different spatial scales. Journal of Experimental Marine Biology and Ecology 216: 77-98.

Holm, S. 1979. A simple sequentially rejective multiple test procedure. Scand. J. Stat. 6: 6570.

Ihaka, R. \& Gentleman, R. 1996. R: a language for data analysis and graphics. Journal of Computational and Graphical Statistics 5: 299-314.

Jelinski, D. E. \& Wu, J. 1996. The modifiable areal unit problem and implications for landscape ecology. Landscape Ecology 11: 129-140.

Jonsson, B. G. \& Moen, J. 1998. Patterns in species associations in plant communities: the importance of scale. Journal of Vegetation Science 9: 327-332. 
1 Juhász-Nagy, P. 1976. Spatial dependence of plant populations. Part I: Equivalence Analysis (an Outline for a New Model). Acta Botanica Academiae Scientiae Hungaricae 22(12) : 61-78.

Juhász-Nagy, P. 1984. Spatial Dependence of Plant Populations. Part 2: A Family of New Models. Acta Botanica Hungarica 30(3-4) : 363-402.

Juhász-Nagy, P. \& Podani, J. 1983. Information theory methods for the study of spatial processes and succession. Vegetatio 51: 129-140.

Kenkel, N. C., Juhasz-Nagy, P. \& Podani, J. 1989. On sampling procedures in population and community ecology. Vegetatio 83: 195-207.

Kershaw, K. A. 1957. The use of cover and frequency in the detection of pattern in plantcommunities. Ecology 38(2): 291-299.

Legendre, P. 1993. Spatial autocorrelation: trouble or new paradigm? Ecology 74(6): 16591673.

Legendre, P. \& Legendre, L. 1998. Numerical ecology. $2^{\text {nd }}$ English ed. Elsevier Science, Amsterdam, NL.

Legendre, P. \& Gallagher, E. D. 2001. Ecologically meaningful transformations for ordination of species data. Oecologia 129: 271-280.

Legendre, P., Dale, M. R. T., Fortin, M-J., Casagrin, P. \& Gurevitch, J. 2004. Effects of spatial structures on the results of field experiments. Ecology 85(12): 3202-3214.

Mistral, M., Buck, O., Meier-Behrmann, D. C., Burnett, D. A., Barnfield, T. E., Scott, A. J., Anderson, B. J. \& Wilson, J. B. 2000. Direct measurement of spatial autocorrelation at the community level in four plant communities. Journal of Vegetation Science 11: 911-916. 
1 Moran, M. D. 2003. Arguments for rejecting the sequential Bonferroni in ecological studies.

2

3 Oikos 100(2): 403-405.

Moran, P. A. P. 1950. Notes on continuous stochastic phenomena. Biometrika 37: 17-23.

Noy-Meir, I. \& Anderson, D. 1971. Multiple pattern analysis or multiscale ordination: towards a vegetation hologram. In: Patil, G. P., Pielou, E.C., and Water, E.W. (eds.). Statistical Ecology: populations, ecosystems, and systems analysis. Vol. 3, pp. 207232. Pennsylvania State University Press, University Park, Pennsylvania, USA.

Openshaw, S. 1984. The modifiable areal unit problem. 38, Norwich, Geo Books.

Otypková, Z. \& Chytry, M. 2006. Effects of plot size on the ordination of vegetation samples. Journal of Vegetation Science 17: 465-472.

Palmer, M. W. 1987. Variability in species richness within Minnesota oldfields: a use of the variance test. Vegetatio 70: 61-64.

Palmer, M. W. 1995. How should one count species? Natural Areas Journal 15: 124-135.

Palmer, M. W. \& White, P. S. 1994. Scale dependence and the species-area relationship. American Naturalist 144: 717-740.

Podani, J. 1984. Spatial Processes in the Analysis of Vegetation: Theory and Review. Acta Botanica Hungarica 30: 75-118.

Podani, J. \& Csontos P. 2006. Quadrat size dependence, spatial autocorrelation and the classification of community data. Community Ecology 7 (1): 117-127.

Reed, R. A., Peet, R. K., Palmer, M. W. \& White, P. S. 1993. Scale dependence of vegetationenvironment correlations; a case study of a North Carolina piedmont woodland. Journal of Vegetation Science 4: 329-340. 
1 Scheller, R. M. \& Mladenoff D. J. 2002. Understory species patterns and diversity in oldgrowth and managed northern hardwood forests. Ecological Applications 12: 13291343.

Seabloom, E. W., Bjornstad, O. N., Bolker, B. M. \& Reichman, O. J. 2005. Spatial signature of environmental heterogeneity, dispersal, and competition in successional grasslands. Ecological Monographs 75(2): 199-214.

Ter Braak, C. J. F. 1983. Principal components biplots and alpha and beta diversity. Ecology 64: 454-462.

Ter Braak, C. J. F. 1995. Ordination. In: Jongman, R.H.G.; Ter Braak, C.J.F. und Van Tongeren, O.F.R. Data analysis in community and landscape ecology. Cambridge University Press, Cambridge.

Ver Hoef, J.M. \& Glenn-Lewin, D.C. 1989. Multiscale ordination: a method for detecting pattern at several scales. Vegetatio 82: 59-67.

Wagner, H. H. 2003. Spatial covariance in plant communities: integrating ordination, geostatistics, and variance testing. Ecology 84(4): 1045-1057.

Wagner, H. H. 2004. Direct multi-scale ordination with canonical correspondence analysis. Ecology 85(2): 342-351.

Wagner, H. H. \& Fortin, M.-J. 2005. Spatial analysis of landscapes: concepts and statistics. Ecology 86(8): 1975-1987.

Waite, S. 2000. Statistical ecology in practice. A guide to analysing environmental and ecological field data. Pearson Education Limited, Harlow.

Wiens, J.A. 1989. Spatial scaling in ecology. Functional Ecology 3: 385-397. 


\section{Tables}

2 Table 1: Conceptual differences between ordination and spatial pattern analysis.

\begin{tabular}{|c|c|c|}
\hline Approach & Gradient analysis (ordination) & Spatial pattern analysis \\
\hline Focus & True gradients & False gradients \\
\hline Design & $\begin{array}{l}\text { Simple or stratified random samples } \\
\text { Regularly spaced samples } \\
\text { Sampling along environmental gradients }\end{array}$ & Transects or grids of contiguous quadrats \\
\hline Environment & Heterogeneous & Homogeneous \\
\hline Observations & Spatially independent & Spatially autocorrelated \\
\hline Target & $\begin{array}{l}\text { Data reduction } \\
\text { Importance of environmental factors }\end{array}$ & $\begin{array}{l}\text { Scaling properties of variance } \\
\text { Strength and spatial scale of internal } \\
\text { organization }\end{array}$ \\
\hline
\end{tabular}

3

\section{Figures}

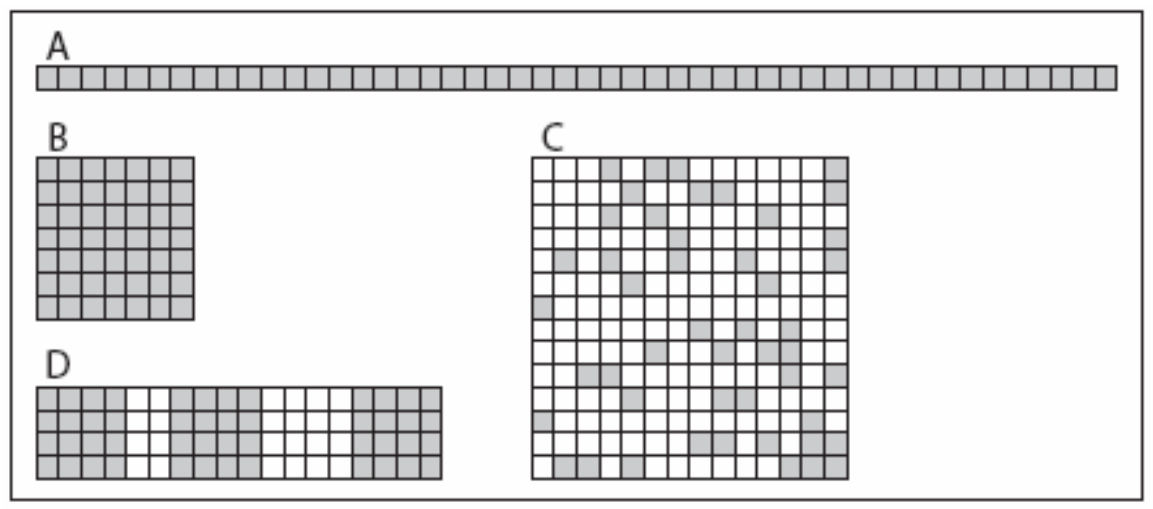

6 Figure 1: Four different sampling designs with comparable sample size: (A) transect of 48

7 continuous quadrats; (B) single block of $7 \times 7$ quadrats; (C) random sample of 48 out of $14 \times$

814 quadrats; and (D) three blocks of $4 \times 4$ quadrats spaced by a distance of 2 and 4 quadrat

9 lengths. 

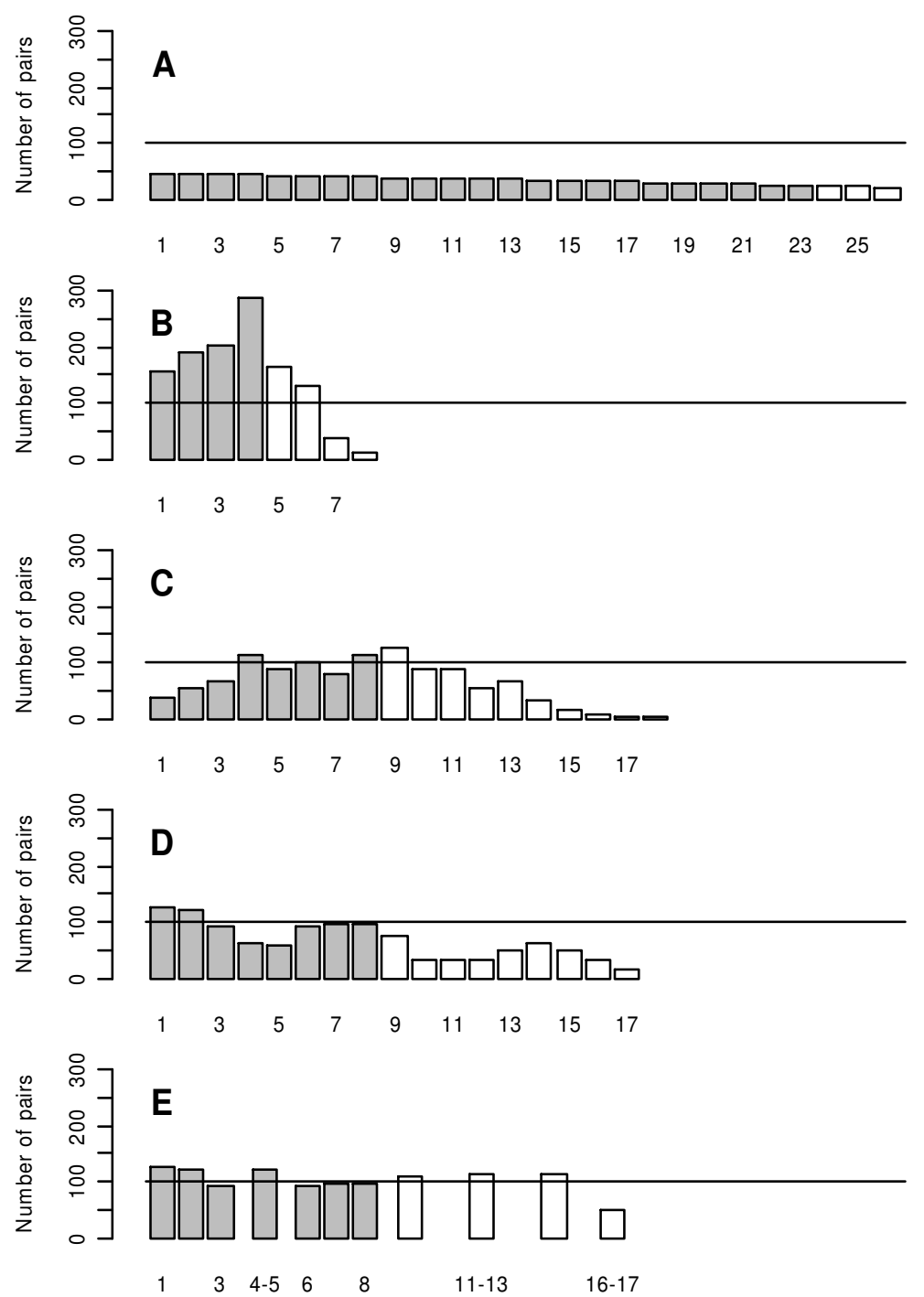

2 Figure 2: Bar plots of the number of pairs of observations per distance class for different

3 sampling designs: transect (A) (only 26 out of 47 distance classes shown), single block (B),

4 random (C), and 3-blocks design without (D) and with pooling (E) of neighbouring distance

5 classes with low numbers of pairs. Grey bars indicate distance classes within the maximum

6 extent for interpretation. 

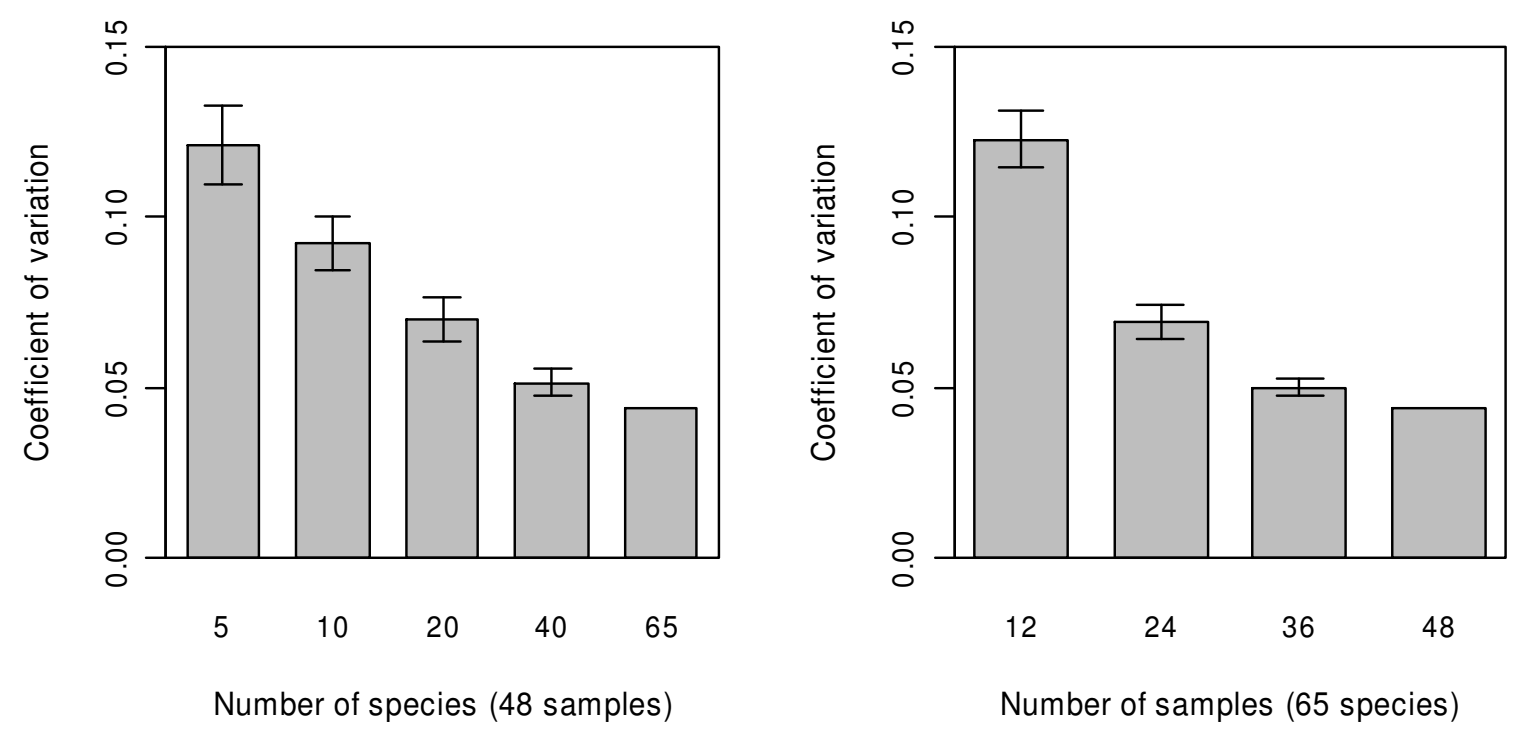

2 Figure 3: Precision of variogram estimates as a function of number of species (left panel) and

3 sample size (right panel) on the basis of the Marchairuz data set. Each bar denotes the mean

$4 \mathrm{CV}$ of variogram estimates for 100 replicate subsamples of species (left panel) or quadrats

5 (right panel), calculated over all non-significant distance classes of each empirical variogram

6 up to half the maximum distance between quadrats. Error bars indicate the $95 \%$ confidence

7 interval for the mean. 

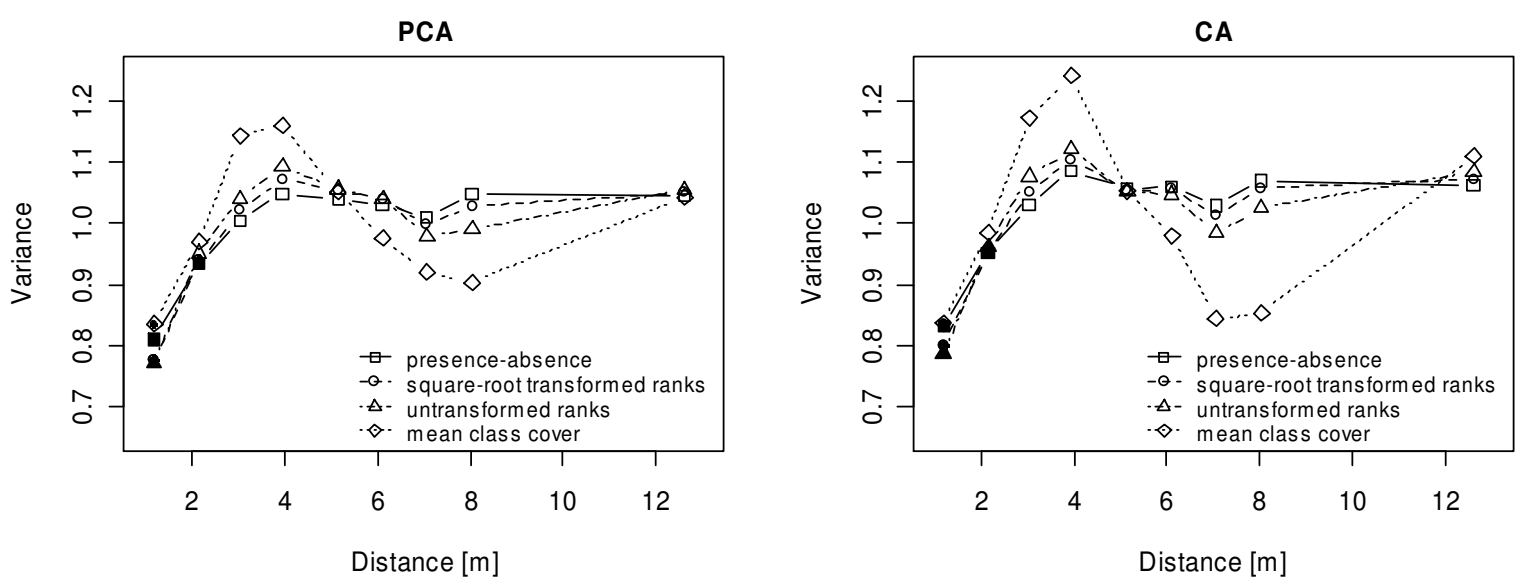

2 Figure 4: Empirical variograms of the total variance on the basis of different data types of the

3 Marchairuz data set based on PCA (left panel) and on CA (right panel). Filled symbols

4 indicate distance classes with positive spatial autocorrelation. All distance classes beyond 8.5

$5 \mathrm{~m}$, the maximum extent for the interpretation of the empirical variogram, were pooled in a

6 single distance class. 

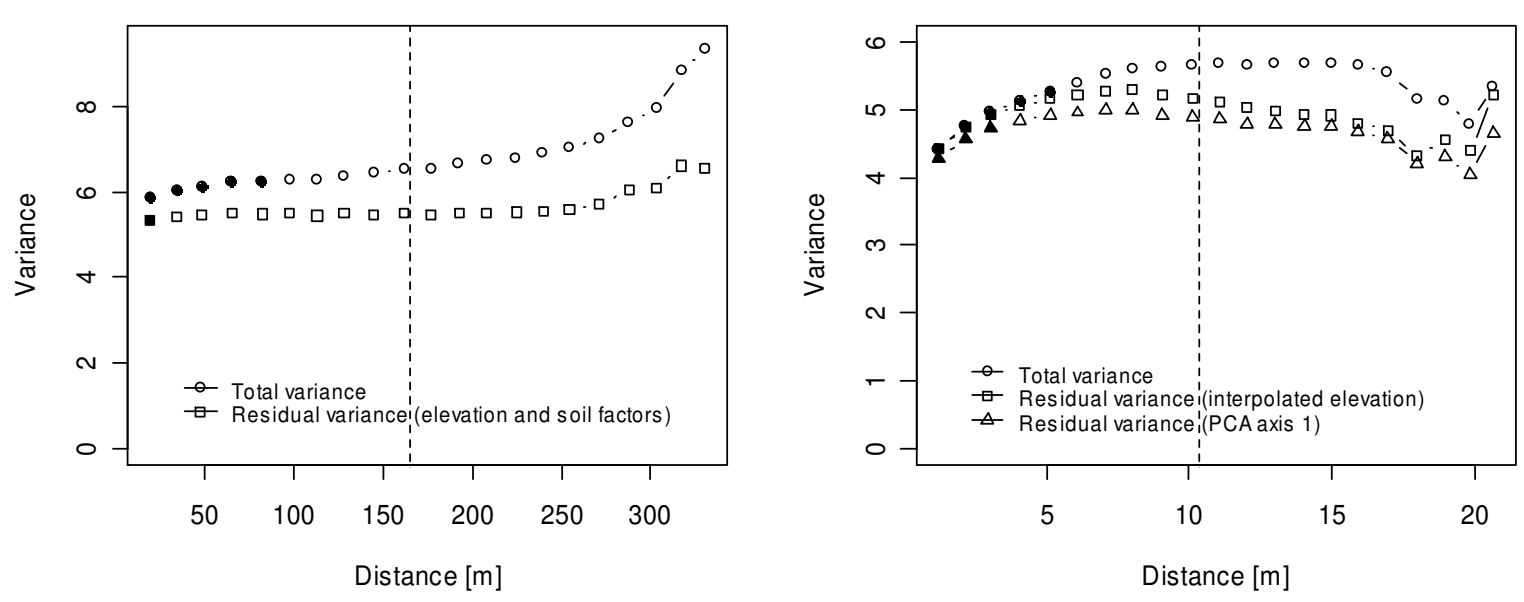

2 Figure 5: Left panel: Empirical variograms of the total variance and residual variance after

3 accounting for explanatory variables of the entire Oosting data set based on PCA and RDA.

4 Right panel: Empirical variograms of the total variance and residual variance after accounting

5 for interpolated elevation or PCA 1 of the SM3 Oosting data set based on PCA and RDA. The

6 maximum extent for the interpretation of the variogram (vertical dashed line) is $165 \mathrm{~m}$ (left)

7 and $10 \mathrm{~m}$ (right). Filled symbols indicate distance classes with positive spatial autocorrelation. 


\section{Appendix}

\section{Multiscale ordination}

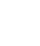

Multiscale ordination was originally proposed by Noy-Meir and Anderson (1971) and further developed by Ver Hoef and Glenn-Lewin (1989) as a method for performing spatial pattern analysis in multi-species communities (Dale 1999). While the original method was based on blocked quadrat variance techniques, Wagner (2003) proposed a geostatistical version that formally integrates ordination based on PCA with multivariate variography. The approach has been generalized to direct ordination with RDA and CCA (Wagner 2004) and to a broad range of ordination methods (Couteron and Ollier 2005).

unbiased variance of a variable $y_{\mathrm{i}}$ observed at $\mathrm{N}$ locations $a$ can be estimated not only from the $\mathrm{N}$ squared deviations from the mean, but equally from the $n$ pairwise differences between observations $a$ and $b$ :

$$
\operatorname{Var}\left(y_{i}\right)=\sum_{a} \frac{\left(y_{i a}-\bar{y}_{i}\right)^{2}}{N-1}=\sum_{a \neq b} \frac{\left(y_{i a}-y_{i b}\right)^{2}}{2 n} \text {, where } n=N(N-1) \text {, }
$$
and similarly for the covariance between two variables $i$ and $j$. The basic elements of spatial covariance,

$$
\gamma_{i j}(a, b)=\frac{1}{2}\left(y_{i a}-y_{i b}\right)\left(y_{j a}-y_{j b}\right)
$$

can be grouped into distance classes $h$ by the geographical distance separating $a$ and $b$, resulting in a set of distance-dependent variance-covariance matrices $\mathbf{C}(\mathrm{h})$ that collectively form the variogram matrix. The weighted average of the variogram matrix is equal to the nonspatial variance-covariance matrix $\mathbf{C}$ : 


$$
\mathbf{C}=\sum_{h} \frac{n_{h}}{n} \mathbf{C}(h)
$$

The variogram matrix contains in the diagonal elements $c_{\mathrm{ii}}(\mathrm{h})$ the variogram of species $\mathrm{i}$, and the off-diagonal cells $c_{\mathrm{ij}}(\mathrm{h})$ the cross-variogram of the two species $i$ and $j$. Plotting the sum of the diagonal against distance provides a variogram of the total variance in the data set, or a community variogram, and plotting the sum of the entire matrix against distance results in a variogram of species richness (for presence-absence data) or of total abundance (for abundance data). The variograms of an individual ordination axis $f$ with associated eigenvector $\mathbf{u}_{\mathrm{f}}$ is derived as the spatial partitioning of its eigenvalue $\lambda_{\mathrm{f}}$ :

$$
\lambda_{f}(h)=\mathbf{u}_{f}^{T} \mathbf{C}(h) \mathbf{u}_{f} .
$$

A worked example is published as a digital appendix to Wagner (2003) and can be found at http://esapubs.org/archive/ecol/E084/023/appendix-A.htm. In direct ordination, separate variograms are derived for the constrained and the residual variance, as implemented in the R library "mso" published as a digital appendix to Wagner (2004) at http://esapubs.org/archive/ecol/E085/006/suppl-1.htm.

\section{Marchairuz data}

The study site was located at $46^{\circ} 32^{\prime} \mathrm{N}, 6^{\circ} 14^{\prime} \mathrm{W}$, at an altitude of $1355 \mathrm{~m}$ a.s.l.

17 Sampling was done in July 2003.

Within three plots of $4 \times 4 \mathrm{~m}, 16$ quadrats of $1 \mathrm{~m}^{2}$ in size were sampled $(\mathrm{N}=48)$. In each quadrat, the cover of vascular plant species was estimated according to the coverabundance classes of Braun Blanquet (1964), which were modified by Dierschke (1994): $r=$ " $<1 \%$, one individual", $+=$ = $<1 \%$, two to five individuals", $1=$ "1-5\%", $2 \mathrm{a}=6-12.5 \%, 2 \mathrm{~b}=$ $12.6-25 \%, 3=26-50 \%, 4=51-75 \%$, and $5=76-100 \%$. Additionally, total cover of lichens 
1 (average of 39.2) but a low cover value of the species. The cover value 1-5\% had the highest

2 frequency with 988 of 1881 cases. The entire data set contained 1239 zeros.

For the main analysis, abundance was quantified as the square-root of ranktransformed Braun Blanquet values (Waite 2000; Gauch 1982), resulting in a scale between 0 and sqrt(8) that balances the weight of presence-absence and dominance information.

6 Correspondence analysis is very sensitive to the presence of species that occur only at a few, species-poor sites (Ter Braak 1995) so that rare species may have a large influence on the analysis (Legendre and Gallagher 2001). Therefore species that occurred only up to three times in the whole data set were excluded from the analysis (Gauch 1982). While infrequent species may be important e.g. for questions relating to species diversity, such rare incidences

11 offer little information for spatial pattern analysis. After removing 8 infrequent species, 65 species remained in the data.

\section{Oosting data}

The study area was situated in the Oosting Natural Area of the Duke Forest, Orange County, North Carolina. It contained several forest communities with gradual transitions. The entire sampling grid contained 256 modules of 16 x $16 \mathrm{~m}$. Within this larger grid, three randomly selected modules were studied more intensively with plant data being collected for every cell of $1 \mathrm{~m}^{2}$. Besides the dataset containing the entire grid, we used module SM3 that had previously been analyzed by Wagner (2003). Four quadrats of side length $0.125 \mathrm{~m}, 0.25$ $\mathrm{m}, 0.5 \mathrm{~m}$, and $1 \mathrm{~m}$ were nested in the southwest corner of each of the 256 contiguous cells. The presence of each vascular plant species was recorded in each quadrat.

Prior to statistical analysis, species that occurred only up to three times were excluded from the analysis (see above). In the order of increasing quadrat size, 20, 22, 25 and 24 species were excluded, so that the final series contained 13, 27, 42 and 62 species. After 
1 removing quadrats without any remaining species, the four series contained 129, 236, 254 and 2256 quadrats.

\section{$3 \quad$ Figures Appendix}
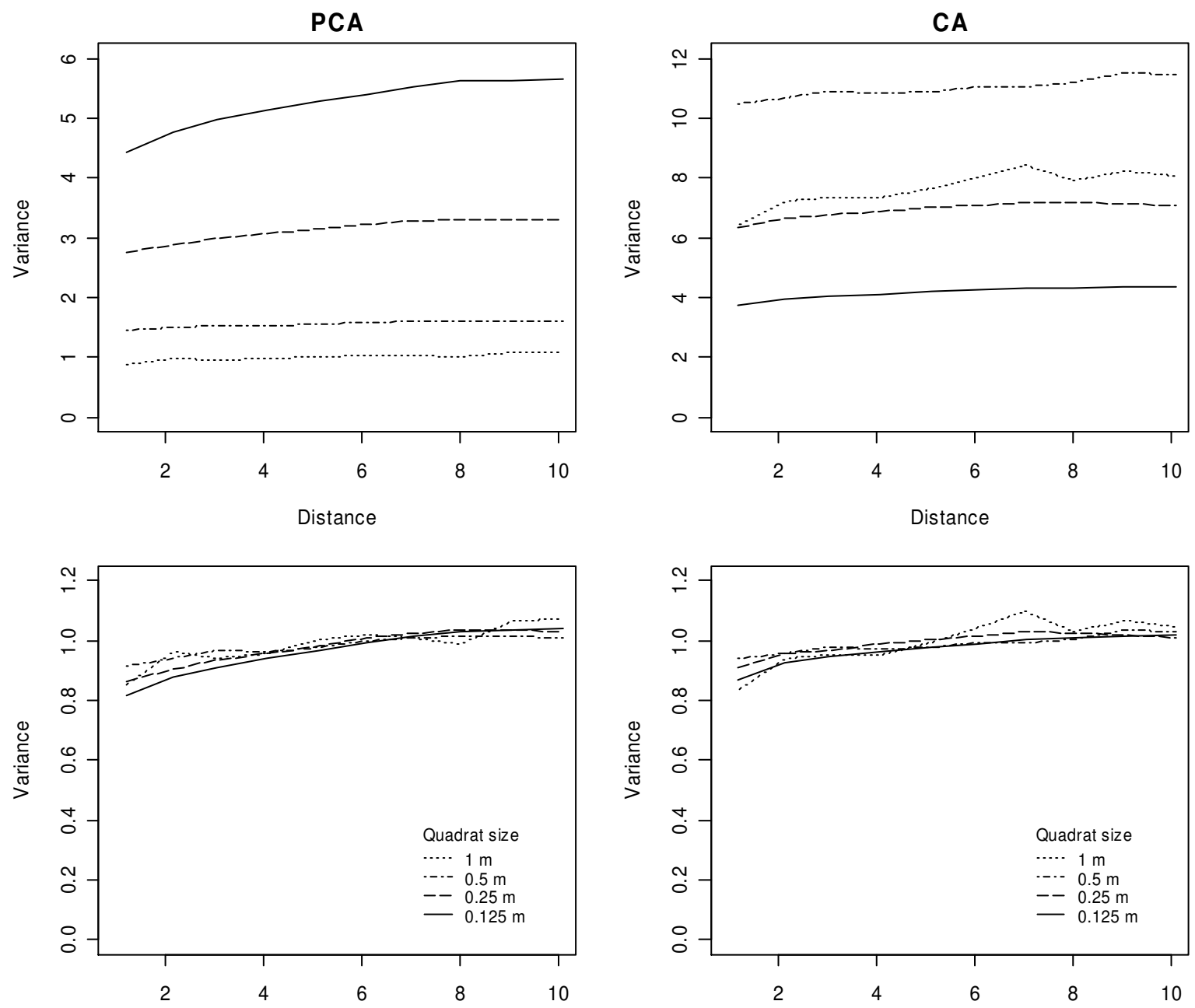

Distance

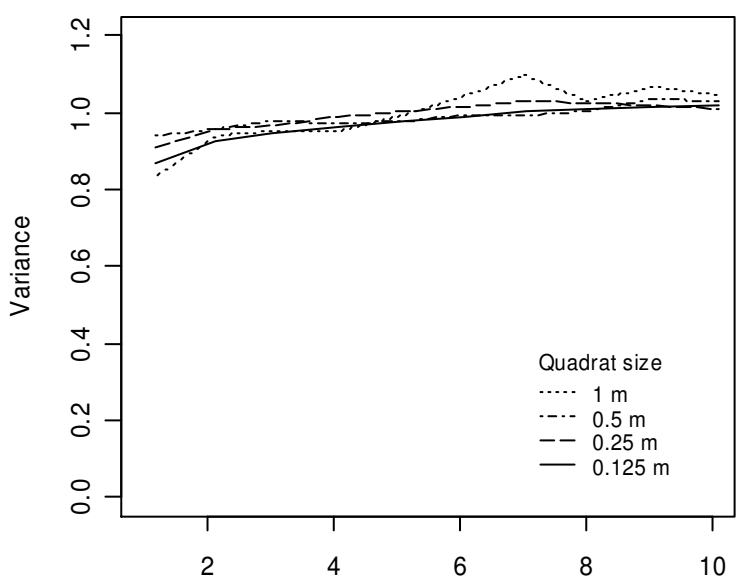

Distance

5 Figure A.1: Effect of quadrat size on the observed spatial pattern on the basis of the Oosting

6 data set. Each line shows an empirical variogram of the total variance (top) or a standardized

7 empirical variogram of the residual variance after accounting for interpolated elevation

8 (bottom) in a PCA (left) and CA (right) framework for a cell size of $1 \mathrm{~m}, 0.5 \mathrm{~m}, 0.25 \mathrm{~m}$ or

$9 \quad 0.125 \mathrm{~m}$. 


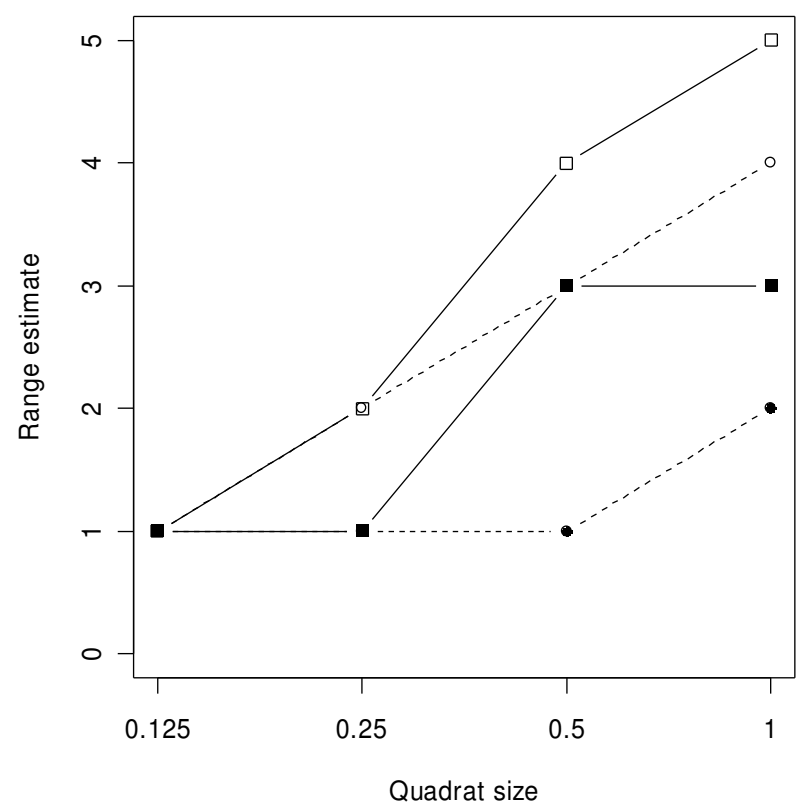

2 Figure A.2: Interaction plot of the effects of quadrat size and ordination method on the

3 estimated range of spatial autocorrelation of the Oosting data set. Each point denotes, for a

4 given quadrat size, the number of consecutive distance classes with significant positive spatial

5 autocorrelation based on a one-sided permutation test with progressive Bonferroni correction

6 based on PCA variograms (empty squares) and CA variograms (empty circles) of total

7 variance, and on RDA (solid squares) and CCA (solid circles) residual variograms. 